\title{
Surgical management of true vaginal prolapse in a Mongrel bitch
}

\author{
S. Ruhil ${ }^{1 *}$, S. Potliya ${ }^{1}$, H. Singh ${ }^{1}$, A. Kumar ${ }^{1}$ and R. S. Bisla ${ }^{1}$ \\ ${ }^{1}$ Haryana Pashu Vigyan Kendra, Lala Lajpat Rai University of Veterinary and Animal Sciences, \\ Uchani, Karnal-132 001, Haryana, India
}

\begin{abstract}
The present study describes the possible etiology, clinical diagnosis and successful surgical management of Type III vaginal prolapse by modified Schaefer's technique. The report justifies the excision of prolapsed mass for correction as well as prevention of recurrence of the prolapse in affected animals.
\end{abstract}

Key words: Dystocia, Estrogen, Mongrel, Prolapse, Surgery

Canine reproductive problems are very challenging for veterinarians. They also contribute significantly to morbidity and infertility in bitches (Anya et al., 2020). Out of various vaginal pathologies like tumours, vaginitis etc., the incidence of true vaginal prolapse is very low (Varudharajan et al., 2020). It has been documented that true vaginal prolapse (TVP) usually occurs near or at parturition (Sarkar et al., 2019). Estrogen and relaxin hormones are the major players in the pathogenesis of true vaginal prolapse. There are breed dispositions for vaginal fold prolapse. It has been frequently reported in brachycephalic breeds and some large breeds like Dalmatians, Doberman (Antonov et al., 2009). But reports on Mongrel are sparse. The majority of the clients hesitate to go for surgical intervention which further complicates the problem (Anya et al., 2020). Hence, the present report describes the successful surgical management of true vaginal prolapse in a Mongrel bitch.

A four-year-old, $25 \mathrm{~kg}$ Mongrel bitch was presented to Teaching Veterinary Clinical Complex (TVCC), Uchani, Karnal, with the primary complaint of a large oedematous mass protruding from vulvar lips since the time of whelping. The bitch had a history of dystocia at parturition around 15 days ago. The animal was active with mild signs of dehydration, had a good appetite but there was difficulty in urination and sitting. All clinical parameters such as rectal temperature, pulse rate and respiration rates were within normal range. The prolapsed mass was washed with mild disinfectant $\left(0.1 \% \quad \mathrm{KMnO}_{4}\right)$. By manual palpation and differential diagnosis with other vaginal pathologies, it was clinically diagnosed as type III vaginal prolapse. It was characterized by complete prolapse of vaginal circumference along with part of the cervix, protruding out from vulvar lips with an appearance like a doughnut (Antonov et al., 2009). Initially, the urinary bladder was catheterized (Romsoms Feeding Tube No. 8) and prolapsed mass was massaged with a mixture of $2 \%$ lignocaine jelly and ointment, Soframycin. Attempts were made to replace it back by applying gentle bilateral pressure from ventral as well as lateral sides (Fig. 1). Being unsuccessful even after many attempts, a modified version of Schaefer's surgical technique (2001) was opted. The animal was administered inj. ceftiofur sodium @ $2.2 \mathrm{mg} /$ $\mathrm{kg}$ body weight $\mathrm{I} / \mathrm{M}$ (Xnel $250 \mathrm{mg}$ ), inj. meloxicam @ $0.5 \mathrm{mg} / \mathrm{kg}$ body weight I/M (Melonex), Inj. B-complex $1 \mathrm{~mL}$ I/M (Polybion), inj. ranitidine @ $0.5 \mathrm{mg} / \mathrm{kg}$ body 
Indian Journal of Animal Health, December, 2021

Vaginal prolapse in Mongrel bitch

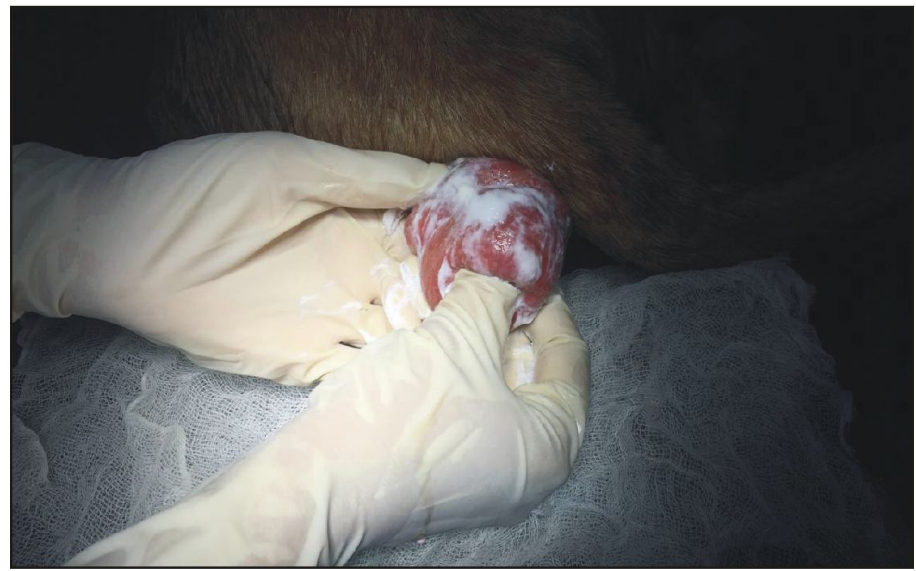

Fig. 1. Repositioning of the prolapsed mass

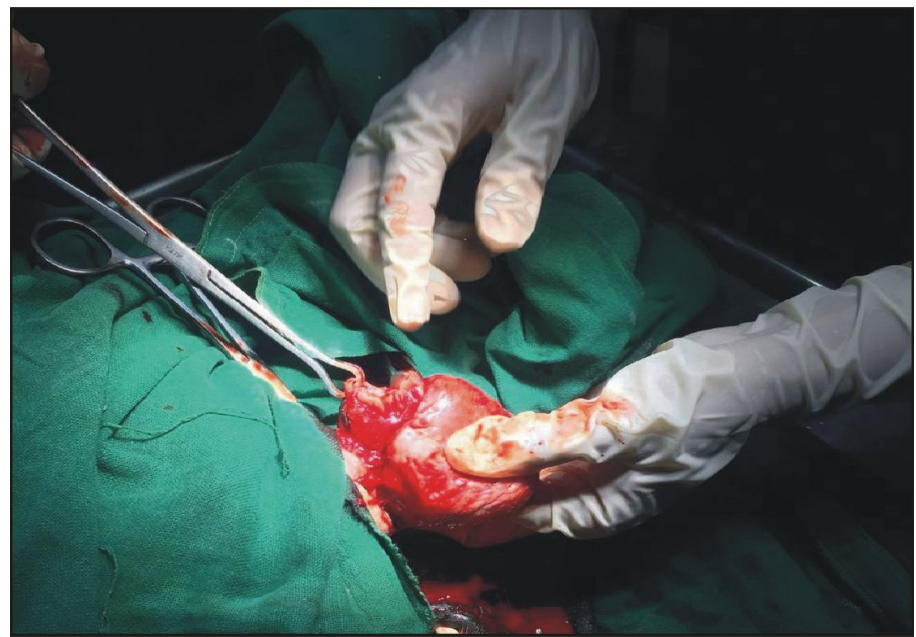

Fig. 2. Excision of prolapsed mass

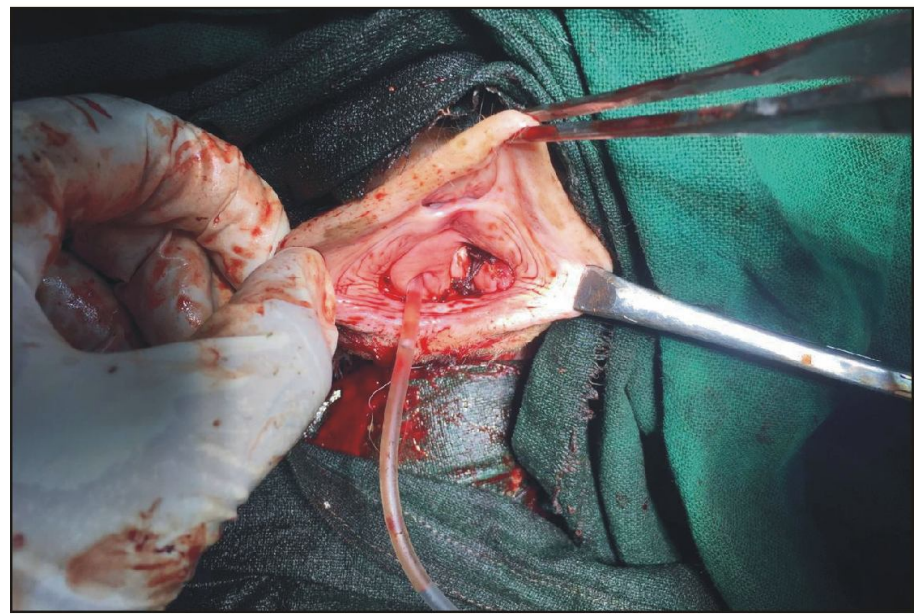

Fig. 3. Releasing of the stump back in the vagina 
weight I/M (Rantac) and was given fluid therapy (Normal saline solution @ 4-6 mL/kg/ $\mathrm{hr}$ and Ringer lactate @ 4-6 mL/kg/hr Intravenously). Thereafter, the animal was premedicated by inj. atropine sulphate @ 0.04 $\mathrm{mg} / \mathrm{kg} \mathrm{S} / \mathrm{C}$ and inj. xylazine hydrochloride @ $0.5 \mathrm{mg} / \mathrm{kg}$ bw I/M (Xylodac). General anaesthesia was induced and subsequently maintained by inj. propofol @ 4-6 mg/kg body weight as I/V bolus till effect (Sarkar et al., 2019). Bitch was placed in dorsal recumbency with endotracheal intubation. A series of horizontal mattress sutures were applied by using absorbable Truglyde suture No. 2 (Sutures India) at the entire circumference and prolapsed mass was excised carefully by electric thermocautery (Fig. 2). The formed stump was replaced back in the vagina after topical application of liq. povidone iodine (Fig. 3). The animal was prescribed supportive therapy along with laxatives for a further five days. Antiseptic dressing of the surgical wound with povidone iodine solution was suggested for at least 10 to 12 days. An Elizabethan collar was set to prevent licking and biting of sutures. The bitch recovered eventually without any complications. Recurrence of prolapsed mass was not reported even after 24 weeks postsurgery.

The present case reports the occurrence of TVP in a Mongrel bitch at the time of whelping. This is in contrast to many reports (Johnston et al., 2001; Varudharajan et al., 2020) which suggest the pure breed predisposition. The probable reason for this occurrence in Mongrel may be the involvement of other predisposing factors apart from genetics. The predisposing factors may include an increase in abdominal

\section{REFERENCES}

Ahuja AK, Dhindsa SS, Kumar A and Singh P, 2018. Surgical approach for vaginal hyperplasia and vaginal fold prolapse in bitch. J Anim Health Prod, 6(3): 86-89, doi:10.17582/journal.jahp/ 2018/6.3.86.89

Antonov AL, Atanassov AS and Georgiev PI, 2009. A pressure due to the presence of fetuses, difficult labor, constipation and excess relaxation of pelvic ligaments along with the cervix under the influence of increasing estrogen as well as relaxin hormone at whelping (Yesilkaya et al., 2020). The exact cause of this condition is still unclear. In accordance with Yesilkaya et al. (2020), the present report depicts TVP in bitch with the history of dystocia. Treatment options vary from conservative management to the surgical removal of prolapsed mass depending upon the degree of prolapse, stage of the reproductive cycle, and breeding value of bitch (Aronson, 2016). In the present study, Type III vaginal prolapse was managed surgically in accordance with Schaefers-Okkens (2001). A modified Schaefer's technique was used because of the limitation to close the vagina until sutures are reabsorbed. The technique described in this report has the advantage of ease of performing, efficient haemostasis as well as offers minimum risk to the neighbouring structures. It has already been used successfully by Ahuja et al. (2018) without any evidence of recurrence.

We concluded that in the present case, the most probable cause of vaginal prolapse was dystocia. The extreme straining during dystocia leads to loss of tonicity of genital organs predisposing the bitch to vaginal prolapse. The present case report suggests that in chronic cases of prolapse, the choice of treatment should be the surgical amputation of prolapsed mass for effective and long-term management without any complications.

Conflict of interest: Authors have no conflict of interest in this study.

modified technique for prolapsed fold excision in a bitch with vaginal hyperplasia. Bulg J Vet Med, 12(4): 260-264

Anya KO, Oguejiofor CF, Nnaji TO and Udeani IJ, 2020. Vaginal hyperplasia and progressive vaginal fold prolapse in a Bullmastiff bitch. Open J Vet Med, 
10(5): 55-63, doi: 10.4236/ojvm.2020.105005

Aronson LR, 2016. Small Animal Surgical Emergencies, $1^{\text {st }}$ edn., Published by John Wiley and Sons, Ltd, Chapter 45- Uterine and vaginal prolapse by Pieter Nelissen, pp 420-427

Johnston SD, Kustritz MVR and Olson PNS, 2001. Canine and Feline Theriogenology, $1^{\text {st }}$ edn., London: W.B. Saunders, pp 225-273

Sarkar D, Chethana DH, Sandeep KH, Mahendra TH, Rudresh GN et al., 2019. A case report: Necrotic cervico-vaginal prolapse with retroflexion of urinary bladder with secondary miasis in a bitch. Pharma Innovation, 8(4): 654-656

Schaefers-Okkens AC, 2001. Vaginal edema and vaginal fold prolapse in the bitch, including surgical management. In: Recent Advances in Small Animal Reproduction. Concannon PW, England G, Verstegen (eds.): International Veterinary Information Service, Ithaca, New York, USA

Varudharajan V, Selvaraju M, Prakash S and Ravikumar K, 2020. Management of true estrual vaginal prolapse in bitches. Haryana Vet, 59(SI): $132-133$

Yesilkaya OF, Ciftei MF, Satilmis F, Parlak K, Alkan H et al., 2020. Vaginal prolapse complicated with urinary bladder retroflexion and colonic herniation in a dog. Vlaams Diergeneeskd Tijdschr, 89(5): 263-268, doi: $10.21825 /$ vdt. V89i5.16952

Received -13.04.2021, Accepted - 21.06.2021, Published - 30.08.2021(Online), 01.12.2021 (Print) Section Editor: Prof. S. K. Nandi, Associate Editor 